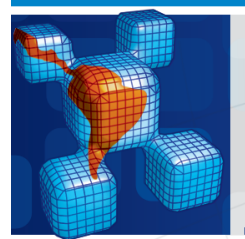

\title{
Acoustic displacement triangle based on the individual element test
}

\begin{abstract}
A three node -displacement based- acoustic element is developed. In order to avoid spurious rotational modes, a higher order stiffness is introduced. This higher order stiffness is developed from an incompatible strain field which computes element volume changes under nodal rotational displacements fields. The higher order strain resulting from the incompatible strain field satisfies the Individual Element Test (IET) requirements without affecting convergence. The higher order stiffness is modulated, element by element, with a factor. As a result, the displacement based formulation presented on this paper is capable of placing the spurious rotational modes above the range of the physical compressional modes that can be accurately calculated by the mesh.

\section{Keywords}

Fluid-structure interaction, Displacement-based formulation, Spurious modes, Individual Element Test, Parameterized Variational Principle
\end{abstract}

\author{
S. Correa*, C. Militello** and \\ M.Recuero ${ }^{* * *}$ \\ * Department of Product Design Engineering. \\ EAFIT University, Medelln-Colombia \\ ${ }^{* *}$ Department of Fundamental Physics, Exper- \\ imental, Electronics and Systems. Universidad \\ de La Laguna, S/C de Tenerife, Spain \\ ${ }^{* * *}$ Department of Mechanics. School of In- \\ dustrial Engineers. Universidad Politecnica de \\ Madrid. Madrid, Spain
}

Received 27 Mar 2012;

In revised form 16 Apr 2012

* Author email: scorrea5@eafit.edu.co

\section{INTRODUCTION}

Acoustic propagation in an inviscid media is generally studied using the pressure $p$ as primitive variable. Consequently, after the finite element approximation, only one unknown variable per node is obtained. This is a drastic reduction on the number of unknown variables as compared to a displacement based formulation where two unknown displacements $u, v$ at each node are necessary to describe the problem. The only drawback in using a pressure formulation can be found when the fluid is interfaced with an elastic solid because both of them do not share the same variables. To overcome this issue, an equilibrium constraint must be imposed at the fluidsolid boundary. Therefore, an acoustic fluid element cannot be handled by a finite element code as any other structural element. Displacement, pressure, displacement-pressure and velocity potential elements have been developed in the past. References $[2-4,7,9,10,12-14]$ are, in the authors' opinion, the most relevant.

Displacement formulations have been reported by many authors. The formulation of these elements following finite element standard procedures produces a rank deficient stiffness matrix. This rank deficiency is not an error because the strain energy in an acoustic fluid is only 
computed from volume changes. The displacement field inside the element computes element shape changes with no volume changes. Any displacement field that describes shape changes without volume variation expands the null space of the stiffness matrix. This displacement field is called spurious because, despite being consistent with the formulation, it is not expected in a true irrotational formulation. Moreover, when an eigenfrequency problem is solved, the spurious displacement field produces low frequency rotational modes. Many authors $[2,10,14]$ compute the rotational of the displacement field and add a fictitious rotational energy. There is not a constitutive equation for such behavior and a fictitious elastic coefficient must be introduced, appearing in the formulation as a penalty factor. Generally, the value suggested for the factor ranges from 1 to 1,000 times the compressibility modulus. This variability of the factor is a serious drawback because the results strongly depend on this a priori tuning.

In this paper, a displacement-based triangular element is proposed. In order to obtain an irrotational displacement field in weak form, an incompatible mode is added. From the added incompatible mode, a higher order strain is computed. After filtering the higher order strain to satisfy the IET from Bergan [5, 11], a higher order stiffness matrix is assembled. No rotational measure is introduced and no fictitious elastic coefficient is needed. However, it is possible to introduce a coefficient in front of the resulting higher order stiffness. The coefficient changes from element to element without affecting convergence. A stabilization strategy is proposed and the coefficient is computed in a closed form as a function of the element size. In this way, the spurious modes are placed over the higher compressional mode that still retains a physical meaning. This formulation is not based in the Raviart-Thomas polynomial [6] nor in the displacement/pressure formulation $[2,14]$, hence no centerface nor midside degrees of freedom are necessary, resulting in lower computational cost.

A 2D acoustic cavity and a 2D fluid interaction problem are presented to show convergence, non uniform meshes behavior and boundary normal definition issues $[2,14]$. The $2 \mathrm{D}$ acoustic cavity is also used to check the appearance of spurious modes.

\section{THE STIFFNESS MATRIX ASSEMBLY}

\subsection{Displacement field and incompatible modes}

To describe the displacement field, the same orientation of the coordinate system is assumed for all the elements. Each element uses a system with origin at its center of gravity. In a linear triangle the general form for the displacement field is:

$$
u_{c}=\left(\begin{array}{l}
u_{c} \\
v_{c}
\end{array}\right)=\left(\begin{array}{c}
a_{1}+a_{2} x+a_{3} y \\
b_{1}+b_{2} x+b_{3} y
\end{array}\right)
$$

Where $\mathbf{u}$ is the displacement field and the subscript $c$ stands for the compatible part. The field is linear and the $a$ and $b$ factors can be obtained as a function of the nodal displacements values $u_{j}, v_{j}$ with $j=1,2,3$ and the nodal coordinates [15].

The following incompatible modes are introduced, 


$$
u_{i}=\left(\begin{array}{c}
u_{i} \\
v_{i}
\end{array}\right)=\left(\begin{array}{c}
\varsigma g(x, y) \\
\eta g(x, y)
\end{array}\right)
$$

Where $\mathbf{u}$ is the displacement field and the subscript $i$ stands for the incompatible part and

$$
g(x, y)=x y^{2}+y x^{2}
$$

Constants $\varsigma$ and $\eta$ must be determined element by element and take values different from zero when the fluid tends to rotate.

A trial displacement field, $u^{*}=u_{c}+u_{i}$ is proposed in order to obtain $\varsigma$ and $\eta$ as a function of the $a$ and $b$ coefficients.

The rotor of the trial field is computed as:

$$
\nabla \times u^{*}=\left(u_{, y}^{*}-v_{, x}^{*}\right)=\left(\left(a_{3}+\varsigma g_{, y}\right)-\left(b_{2}+\eta g_{, x}\right)\right)
$$

From (4), it becomes clear that asking the rotor to cancel at each point inside the element will not produce the sufficient conditions. Instead, the following weak form is tried:

$$
\int_{\Omega_{e}}\left(x^{2}+y^{2}\right)\left(u_{, y}^{*}-v_{, x}^{*}\right) d \Omega=0
$$

By integrating and rearranging terms, (5) results in the following matrix relationship:

$$
\left(\begin{array}{ll}
f_{11} & f_{12} \\
f_{21} & f_{22}
\end{array}\right)\left(\begin{array}{c}
\varsigma \\
\eta
\end{array}\right)=\left(\begin{array}{ll}
p_{11} & p_{12} \\
p_{21} & p_{22}
\end{array}\right)\left(\begin{array}{l}
a_{3} \\
b_{2}
\end{array}\right)
$$

Which, in compact form, becomes:

$$
\mathrm{F} \psi=\mathrm{Pd}
$$

Where $\psi$ and $\mathbf{d}$ are vectors and the terms of matrix $\mathbf{F}$ are:

$$
\begin{aligned}
& f_{11}=\int_{\Omega e} y^{2}\left(2 y x+x^{2}\right) d \Omega \\
& f_{12}=-\int_{\Omega e} y^{2}\left(y^{2}+2 y x\right) d \Omega \\
& f_{21}=\int_{\Omega e} x^{2}\left(2 y x+x^{2}\right) d \Omega \\
& f_{22}=-\int_{\Omega e} x^{2}\left(y^{2}+2 y x\right) d \Omega
\end{aligned}
$$

And the terms of matrix $\mathbf{P}$ are:

$$
\begin{aligned}
& p_{11}=\int_{A_{e}} x^{2} d A \\
& p_{12}=-\int_{A_{e}} x^{2} d A \\
& p_{13}=\int_{A_{e}} y^{2} d A \\
& p_{14}=-\int_{A_{e}} y^{2} d A
\end{aligned}
$$


By nodal collocation, a linear relationship can be obtained between vector $\mathbf{d}$ and the nodal displacements $\mathbf{v}$ :

$$
\mathrm{d}=\mathrm{Q} \mathrm{v}
$$

Where matrix $\mathbf{Q}$ is:

$$
\mathrm{Q}=\frac{1}{\operatorname{det}[\mathrm{J}]}\left[\begin{array}{cccccc}
x_{32} & 0 & x_{13} & 0 & x_{21} & 0 \\
0 & y_{23} & 0 & y_{31} & 0 & y_{12}
\end{array}\right]
$$

Being $x_{i}, y_{i}$ the nodal coordinates and $v^{t}=\left(\begin{array}{lllll}u_{1} & v_{1} & u_{2} & v_{2} & u_{3} \\ v_{3}\end{array}\right)$

Assuming $\mathbf{F}^{-1}$ exists, equation (6) shows that $\psi$ is a null vector for nodal displacements that produces volume changes because it is independent from $a_{2}$ and $b_{3}$, and $\psi \neq 0$ for rotational displacements fields. Thus, rotational fields activate the incompatible modes.

For simplicity, replacing (10) in (7):

$$
\psi=\mathrm{F}^{-1} \mathrm{P} \mathrm{Qv}=\mathrm{Rv}
$$

\subsection{The strain measure.}

In a displacement based acoustic element the only strain measure is the unitary change of volume:

$$
e=u_{, x}+v_{, y}
$$

The pressure inside the element is computed as:

$$
p=\beta e
$$

where $\beta$ is the compressibility modulus of the fluid. In (14) the continuous mechanics convention is used, i.e., a negative change of volume is associated with a negative pressure (stress).

For the element strain two contributions are considered, one from the compatible part of the displacement, $e_{c}$, and a higher order one, $e_{h}$, computed from the incompatible modes $u_{i}$. The computation of $e_{h}$ is not simple because it follows the rules presented in [11], in order to satisfy the IET.

First, a unitary volume change is computed from the incompatible modes:

$$
e_{i}=\varsigma g_{, x}+\eta g_{, y}=\left(\begin{array}{ll}
g_{, x} & g_{, y}
\end{array}\right)\left(\begin{array}{c}
\varsigma \\
\eta
\end{array}\right)=\mathrm{G} \psi
$$

The mean volume averaged strain is computed as:

$$
\bar{e}_{i}=\frac{1}{\Omega_{e}} \int_{\Omega_{e}}\left(\begin{array}{cc}
g_{, x} & g_{, y}
\end{array}\right) d \Omega \psi=\overline{\mathrm{G}} \psi
$$

By subtracting (16) from (15) and replacing (12), the higher order strain field is obtained:

$$
e_{h}=e_{i}-\bar{e}_{i}=(\mathrm{G}-\overline{\mathrm{G}}) \psi=(\mathrm{G}-\overline{\mathrm{G}}) \mathrm{Rv}
$$


This, in a more standard notation, becomes:

$$
e_{h}=(\mathrm{G}-\overline{\mathrm{G}}) \mathrm{Rv}=\mathrm{B}_{\mathrm{h}} \mathrm{v}
$$

From which the higher order stiffness can be computed as:

$$
\mathrm{K}_{\mathrm{h}}=\int_{\Omega_{\mathrm{e}}} \mathrm{B}_{\mathrm{h}}^{\mathrm{t}} \beta \mathrm{B}_{\mathrm{h}} \mathrm{d} \Omega
$$

This higher order stiffness matrix maintains the irrotationality of the fluid. Additionally, the basic stiffness computes the constant strain state, in this case a change of volume, and produces zero energy under rigid body motions, i.e., translation and rotation. This is achieved using $\mathbf{u}_{\mathbf{c}}$ in the more usual shape function expansion:

$$
\mathrm{u}_{\mathrm{c}}=\mathrm{N}^{\mathrm{t}} \mathrm{v}
$$

From this displacement field, the basic strain field is defined as:

$$
e_{b}=\left(\begin{array}{lllll}
N_{1, x} & N_{1, y} & \ldots & N_{3, x} & N_{3, y}
\end{array}\right) \mathrm{v}=\mathrm{B}_{\mathrm{b}} \mathrm{v},
$$

and the basic stiffness is computed,

$$
\mathrm{K}_{\mathrm{b}}=\int_{\Omega_{\mathrm{e}}} \mathrm{B}_{\mathrm{b}}^{\mathrm{t}} \beta \mathrm{B}_{\mathrm{b}} \mathrm{d} \Omega
$$

The total element stiffness is calculated by adding the basic and higher order contributions:

$$
\mathrm{K}_{\mathrm{e}}=\mathrm{K}_{\mathrm{b}}+\alpha \mathrm{K}_{\mathrm{h}}
$$

The value of $\alpha$ can be changed from element to element without affecting the capability of the assembly to correctly define a constant strain state and rigid body modes [8].

\section{STABILIZATION PROCEDURE}

As mentioned in the introduction, one of the disadvantages of the previous formulations is the selection of the penalty factor $\alpha$. A low factor will contaminate the correct modes, whereas a high factor will conceal the contribution of the basic stiffness.

Considering that the basic stiffness computes the irrotational modes from a linear displacement field, it seems reasonable to assume that three elements in a line is the limit to correctly capture half the shortest wavelength. Under these circumstances, it is proposed that the energy computed for a rotational mode of the same wavelength should be of the same order as the energy computed for an irrotational mode. In order to make both energies comparable the eigenfrecuencies are asked to match. The displacement fields that produce rotational and irrotational modes are defined as:

$$
\mathrm{u}_{\mathrm{ir}}^{\mathrm{t}}=\left(\begin{array}{ll}
u_{i r} & v_{i r}
\end{array}\right)=\left(\begin{array}{ll}
\sin \left(\frac{\pi x}{\lambda}\right) & 0
\end{array}\right)
$$




$$
\mathrm{u}_{\mathrm{r}}=\left(\begin{array}{c}
u_{r} \\
v_{r}
\end{array}\right)=\left(\begin{array}{c}
\sin \left(\frac{\pi x}{\lambda}\right) \cos \left(\frac{\pi y}{\lambda}\right) \\
-\sin \left(\frac{\pi y}{\lambda}\right) \cos \left(\frac{\pi x}{\lambda}\right)
\end{array}\right)
$$

Where subscript ir refers to the irrotational displacement field and subscript $r$ refers to the rotational displacement field. These fields satisfy $\nabla \times \mathrm{u}_{\mathrm{ir}}=0$ and $\nabla \cdot \mathrm{u}_{\mathrm{r}}=0$.

To compute the value of $\alpha$ in (23) a regular mesh, as shown in Figure 1, is used. The displacement fields are computed at the mesh nodes and the values are arranged in vectors $\mathbf{U}_{\text {ir }}$ and $\mathbf{U}_{\mathbf{r}}$.

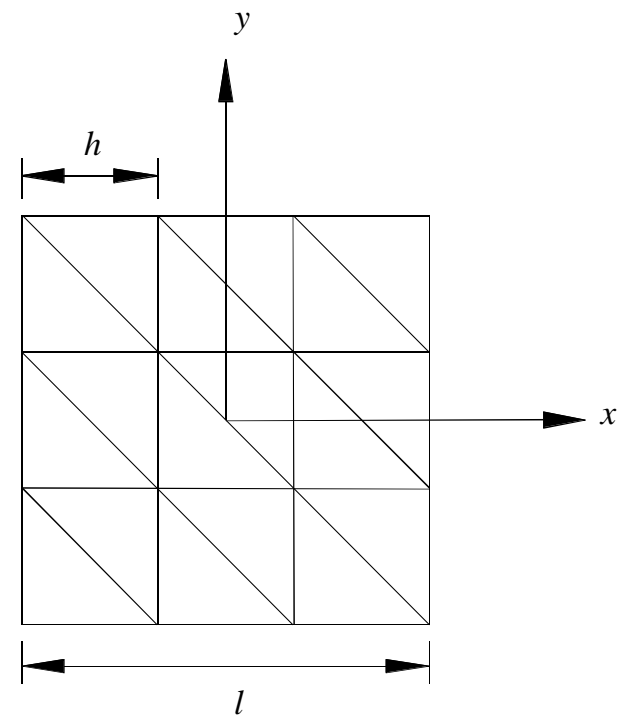

Figure 1 Sample surface mesh used to compute stabilization coefficient $\alpha$.

The approximation to the eigenvalue $(\omega)$ is obtained from the Rayleigh quotient. In both cases, a lumped diagonal mass matrix $(\mathbf{M})$ is used.

$$
\omega_{i r}^{2}=\frac{\mathrm{U}_{\mathrm{ir}}^{\mathrm{T}} \mathrm{K}_{\mathrm{b}}^{\mathrm{a}} \mathrm{U}_{\mathrm{ir}}}{\mathrm{U}_{\mathrm{ir}}^{\mathrm{T}} \mathrm{M}^{\mathrm{a}} \mathrm{U}_{\mathrm{ir}}}
$$

$$
\omega_{r}^{2}=\alpha \frac{\mathrm{U}_{\mathrm{r}}^{\mathrm{T}} \mathrm{K}_{\mathrm{h}}^{\mathrm{a}} \mathrm{U}_{\mathrm{r}}}{\mathrm{U}_{\mathrm{r}}^{\mathrm{T}} \mathrm{M}^{\mathrm{a}} \mathrm{U}_{\mathrm{r}}}
$$

The superscript $a$ indicates the use of the assembled element stiffness matrix from Figure 1. It should be noted that the irrotational field expands the null space of the higher order stiffness matrix, therefore $\alpha$ does not contribute to equation (26). On the other hand, the rotational field expands the null space of the basic stiffness matrix without contributing to equation (27). By equaling both frequencies the following expression for $\alpha$ is obtained:

$$
\alpha=\frac{\mathrm{U}_{\mathrm{ir}}^{\mathrm{t}} \mathrm{K}_{\mathrm{b}}^{\mathrm{a}} \mathrm{U}_{\mathrm{ir}}}{\mathrm{U}_{\mathrm{r}}^{\mathrm{t}} \mathrm{K}_{\mathrm{h}}^{\mathrm{a}} \mathrm{U}_{\mathrm{r}}} \frac{\mathrm{U}_{\mathrm{r}}^{\mathrm{t}} \mathrm{M}^{\mathrm{a}} \mathrm{U}_{\mathrm{r}}}{\mathrm{U}_{\mathrm{ir}}^{\mathrm{t}} \mathrm{M}^{\mathrm{a}} \mathrm{U}_{\mathrm{ir}}}
$$

Latin American Journal of Solids and Structures 2(2012) 1 - 12 
The value of $\alpha$ is independent from physical fluid properties. Equation (28) is computed from the mesh shown in Figure 1. The quadrilateral side is $\lambda / 2$, being $\lambda$ the wavelength, and the relation between quadrilateral side and element side $(h)$ satisfies:

$$
\frac{\lambda}{2}=3 h
$$

Quadrilaterals are constructed for values of $h$ ranging from 0.01 to 10 meters. Rotational and irrotational fields for the corresponding $\lambda$ are computed at the mesh nodes. Finally, equation (28) is evaluated. Hence, a value of $\alpha$ is computed for each value of $h$.

The adjusted function for the pairs $(\alpha, h)$, for $h$ in meters is:

$$
\alpha=\frac{12.9}{h^{2}}
$$

Equation (30) is implemented in the finite element code and is calculated for each element. To compute the element size $h$, the diameter of the circle inscribed in the triangle is used. This method for computing $\alpha$ is conservative because the diameter of the circle is always smaller than the smallest triangle side.

\section{NUMERICAL RESULTS}

The new formulated element is used to solve two 2D problems in order to assess its performance. One consists of a closed rectangular cavity and the other consists of a closed cavity with a skewed corner and a rigid moving piston. In both cases, convergence of the solution varying the element size of the mesh is presented. Since the definition of the normal direction in the solid - fluid interface is critical when imposing impenetrability conditions, the problem of a skewed cavity with moveable piston is also solved for a random variation of that normal direction. To test convergence, four finite element meshes are tried. The first three have one predominant element size and the fourth one has three predominant element sizes in order to demonstrate that the results do not depend on maintaining a constant element size.

\section{CLOSED RECTANGULAR CAVITY.}

When a fluid-structure interaction problem is solved using the displacement based proposed element, it is necessary to impose the impenetrability condition in the interface. For the closed rectangular cavity problem discussed here, the condition is imposed directly in the fluid element restraining the displacement perpendicular to the rigid wall.

The fluid properties and dimensions are shown in Figure 2. The eigenfrequencies can be computed from

$$
f=c \pi \sqrt{\left(\left(\frac{l}{\mathrm{a}}\right)^{2}+\left(\frac{m}{\mathrm{~b}}\right)^{2}\right)}
$$

Where $f$ is the frequency in $[\mathrm{Hz}], c$ is the speed of sound in water $[\mathrm{m} / \mathrm{s}], \mathrm{a}$ is the width in $[\mathrm{m}], \mathrm{b}$ is the height in $[\mathrm{m}], l$ and $m=0,1,2, \ldots$ 


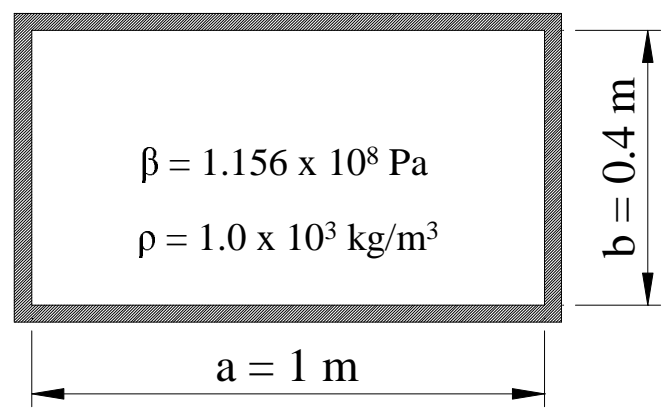

Figure 2 Rectangular cavity. Dimensions and fluid properties.

Four meshes are presented in Figure 3 (left). The first three have one predominant element size (coarse, medium and fine) and the fourth one has three predominant element sizes in the same mesh. The modal pressure distribution for mode 1 is shown at the right side. In Table 1 , the convergence to the first four modes is shown.

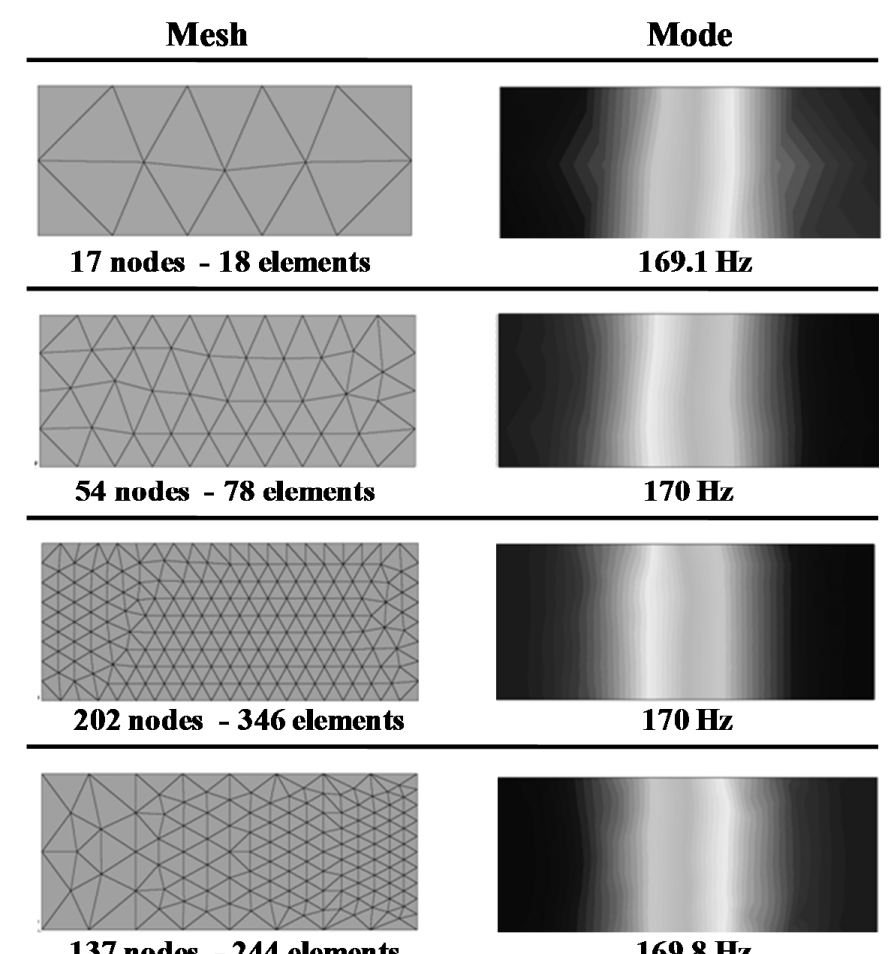

Figure 3 Rectangular cavity results. Meshes at left. Pressure distribution for the $1^{\text {th }}$ mode at right.

\subsection{Spurious mode placement}

According to the stabilization procedure proposed and noting that the coarse mesh from Figure 3 has only two elements in the vertical direction, the first spurious mode must appear after

Latin American Journal of Solids and Structures 2(2012) 1 - 12 
Table 1 Resonant frequencies for a room with rigid walls. Convergence analysis

\begin{tabular}{lllll}
\hline $\begin{array}{l}\text { Mesh } \\
\text { nodes }\end{array}$ & $\begin{array}{l}\text { 1st mode } \\
\text { f= } \mathbf{1 7 0} \mathbf{~ H z}\end{array}$ & $\begin{array}{l}\text { 2nd mode } \\
\mathbf{f}=\mathbf{3 4 0} \mathbf{H z}\end{array}$ & $\begin{array}{l}\text { 3rd mode } \\
\mathbf{f}=\mathbf{4 2 5} \mathbf{H z}\end{array}$ & $\begin{array}{l}\mathbf{4 t h} \text { mode } \\
\mathbf{f}=\mathbf{4 5 7 . 7} \mathbf{H z}\end{array}$ \\
\hline 17 & 169.1 & 350.9 & 384.1 & 478.1 \\
54 & 170 & 341.3 & 421 & 470.5 \\
202 & 170 & 340.3 & 423.3 & 463.7 \\
137 & 169.8 & 340.2 & 430.3 & 459.5 \\
\hline
\end{tabular}

the first half wavelength in that direction. Figures $4 \mathrm{a}$ and $4 \mathrm{~b}$ show the first correct modes in the longitudinal and vertical direction respectively. Figures $4 \mathrm{c}$ and $4 \mathrm{~d}$ show modes with a high tendency to rotate, i.e., these are incorrect (spurious) modes. Also note that the mode in $4 \mathrm{~b}$ is the last one to be correctly calculated using three elements in a half wavelength. In this way, the spurious modes can be located at a convenient place, i.e., with an eigenfrequency higher than the last one that can be adequately calculated by the mesh.

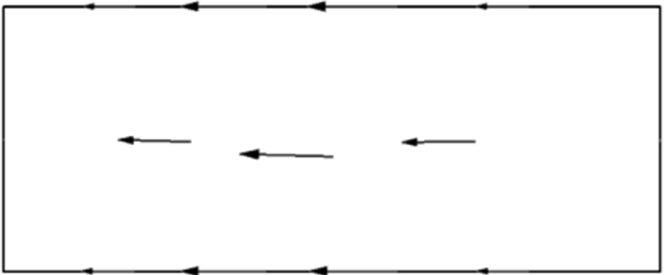

(a)

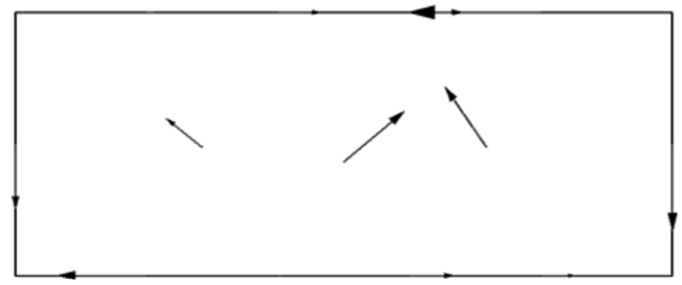

(c)

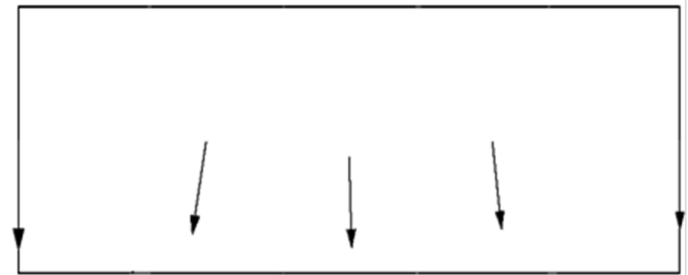

(b)

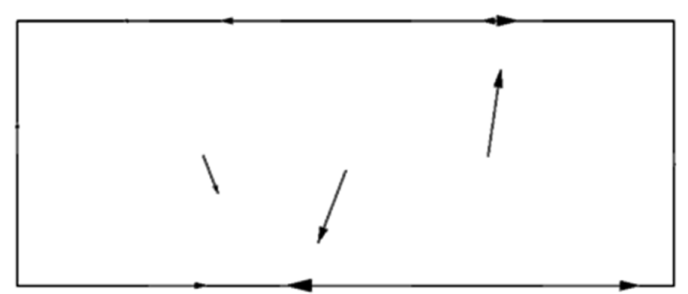

(d)

Figure 4 Spurious modes appearance in a rectangular cavity. (a) First longitudinal mode.( b) First vertical mode $\left(3^{r d}\right.$ mode). (c-d) $5^{\text {th }}$ and $6^{\text {th }}$ modes with a high rotational tendency.

\subsection{Element size sensitivity}

It is necessary to test the sensitivity of the proposed formulation for element sizes $(h)$ out of the range of equation 30 (0.01 to $10 \mathrm{~m}$.). In consequence, two meshes with predominant element sizes of $0.001 \mathrm{~m}$. and $100 \mathrm{~m}$. respectively and having the same number of nodes and elements are tried. The results are summarized in Table 2. 
Table 2 Resonant frequencies for a room with rigid walls. Element size sensitivity

\begin{tabular}{|c|c|c|c|c|c|c|}
\hline $\begin{array}{l}\text { Mesh } \\
\text { nodes }\end{array}$ & $\mathbf{h}[\mathbf{m}]$ & $\alpha$ & $\begin{array}{l}\text { 1st mode } \\
\mathrm{f}=170 \mathrm{~Hz}\end{array}$ & $\begin{array}{l}\text { 2nd mode } \\
\mathrm{f}=340 \mathrm{~Hz}\end{array}$ & $\begin{array}{l}\text { 3rd mode } \\
\mathrm{f}=425 \mathrm{~Hz}\end{array}$ & $\begin{array}{l}\text { 4th mode } \\
\mathrm{f}=457.7 \mathrm{~Hz}\end{array}$ \\
\hline \multirow{2}{*}{202} & 0.001 & $12.9 \times 10^{6}$ & 170 & 340.8 & 423.9 & 464.1 \\
\hline & 100 & $12.9 \times 10^{-4}$ & 170 & 340 & 423.1 & 463.4 \\
\hline
\end{tabular}

\section{SKEWED CAVITY WITH RIGID MOVABLE PISTON.}

A common fluid-structure interaction problem [2], consisting of a skewed rigid piston capable of moving back and forth is presented in Figure 5. For such problem, the impenetrability condition is imposed directly in the fluid element restraining the displacement perpendicular to the rigid wall. Furthermore, the nodes in the piston-fluid interface are forced to move together via Lagrange multipliers.

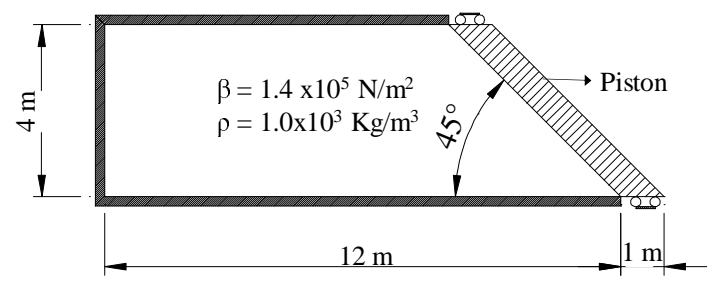

Figure 5 Skewed cavity coupled with movable piston.

Figure 6 shows the meshes and the results obtained for the $3^{\text {rd }}$ and $4^{\text {th }}$ modes.

In Table 3, the convergence of the first four modes is shown. A coarse mesh captures a vertical and a longitudinal mode respectively due to a lack of convergence. A fine mesh captures the correct modes [2] that result from a linear combination of the previous ones. The eigensolver is Eispack through the Matlab interface [1].

Table 3 Resonant frequencies for the skew cavity with rigid piston. Convergence analysis

\begin{tabular}{lllll}
\hline $\begin{array}{l}\text { Mesh } \\
\text { nodes }\end{array}$ & $\begin{array}{l}\text { 1st mode } \\
\mathbf{f = 0 . 2 9} \mathbf{~ H z}\end{array}$ & $\begin{array}{l}\text { 2nd mode } \\
\mathbf{f = 0 . 8 8} \mathbf{~ H z}\end{array}$ & $\begin{array}{l}\text { 3rd mode } \\
\mathbf{f = 1 . 4 5} \mathbf{~ H z}\end{array}$ & $\begin{array}{l}\text { 4th mode } \\
\mathbf{f}=\mathbf{1 . 4 8} \mathbf{~ H z}\end{array}$ \\
\hline 114 & 0.29 & 0.86 & 1.36 & 1.41 \\
498 & 0.29 & 0.87 & 1.44 & 1.48 \\
2362 & 0.29 & 0.88 & 1.45 & 1.48 \\
1358 & 0.29 & 0.90 & 1.46 & 1.48 \\
\hline
\end{tabular}

For the piston-fluid interface, the normal direction is randomly varied \pm 5 degrees. The effect in the convergence is shown in Table 4.

Latin American Journal of Solids and Structures 2(2012) $1-12$ 


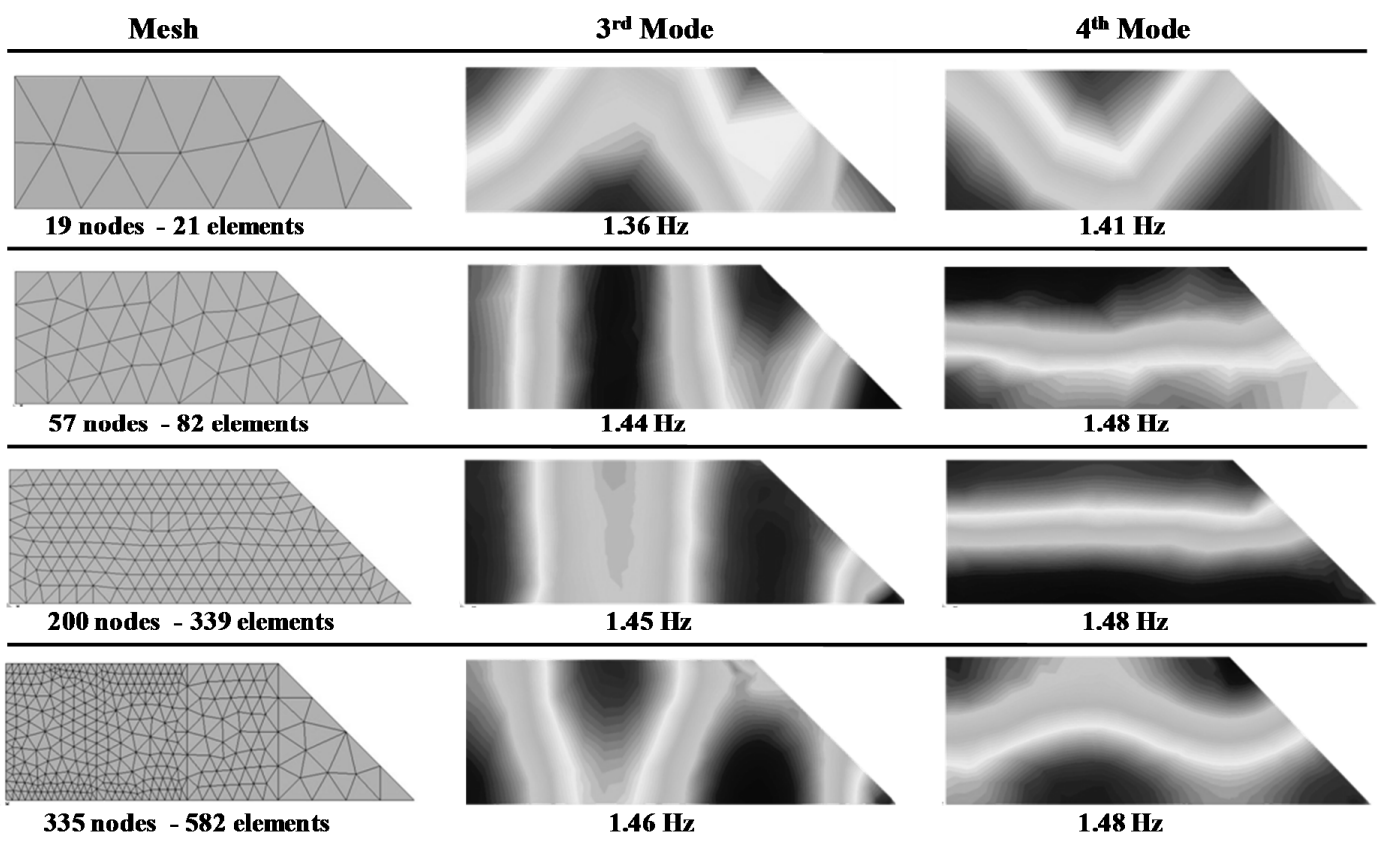

Figure 6 Skew cavity convergence analysis.

Table 4 Resonant frequencies for the skew cavity with rigid piston. Convergence analysis with \pm 5 randomly changed interface normal directions.

\begin{tabular}{lllll}
\hline $\begin{array}{l}\text { Mesh } \\
\text { nodes }\end{array}$ & $\begin{array}{l}\text { 1st mode } \\
\mathbf{f = 0 . 2 9} \mathbf{~ H z}\end{array}$ & $\begin{array}{l}\text { 2nd mode } \\
\mathbf{f = 0 . 8 8} \mathbf{~ H z}\end{array}$ & $\begin{array}{l}\text { 3rd mode } \\
\mathbf{f = 1 . 4 5} \mathbf{~ H z}\end{array}$ & $\begin{array}{l}\text { 4th mode } \\
\mathbf{f}=\mathbf{1 . 4 8} \mathbf{~ H z}\end{array}$ \\
\hline 114 & 0.29 & 0.86 & 1.37 & 1.41 \\
498 & 0.29 & 0.87 & 1.44 & 1.48 \\
2362 & 0.29 & 0.88 & 1.45 & 1.47 \\
1358 & 0.29 & 0.89 & 1.45 & 1.5 \\
\hline
\end{tabular}

\section{DISCUSSION AND CONCLUSIONS.}

The element proposed is a $2 \mathrm{D}$ linear triangle with degrees of freedom that can be easily coupled to $2 \mathrm{D}$ solid elements. Although it has a penalty factor, the proposed energy balance formulation can be implemented directly in the finite element code without user intervention. The penalty factor depends on the element size and does not influence the element convergence. This results in a clear advantage with respect to other fluid-structure interaction elements in which the factor must be selected by the user $[2,10,14]$ and can vary between 1 and 1,000 times the compressibility modulus of the fluid.

The convergence is not altered by a reasonable error in the normal direction definition. The spurious modes do not disappear, as in previous formulations $[2,6,10,14]$. Instead, the penalty factor places spurious modes in frequencies higher than the ones corresponding to the last compression mode accurately calculated by the mesh. 


\section{References}

[1] Matlab. the language of technical computing. 2002. Version 6.5.

[2] K.J. Bathe, C. Nitikitpaiboon, and X. Wang. A mixed displacement-based finite element formulation for acoustic fluid-structure interaction. Computers and Structures, 56:225-237, 1995.

[3] T.B. Belytschko. Fluid-structure interaction. Computer and Structures, 12:459-469, 1980.

[4] T.B. Belytschko and J.M. Kennedy. A fluid-structure finite element method for the analysis of reactor safety problems. Nuclear engineering Design, 38:71-81, 1976.

[5] P.G. Bergan and L. Hanssen. A new approach for deriving good finite elements. in: Jr whiteman. The Mathematics of the Finite Element, 2, 1975.

[6] A. Bermudez and A. Rodriguez. Finite element computation of the vibration modes of a fluid-soil system. Comp. Meth. in Applied Mech. Engineering., 119:355-370, 1994.

[7] G.C. Everstine. A symmetric potential formulation for fluid-structure interaction. Journal of Sound and Vibration, 79:157-160, 1981.

[8] C.A. Felippa and C. Militello. Variational formulation of high performance finite elements: Parametrized variational principles. Computers and Structures, 36:1-11, 1990.

[9] C.A. Felippa and R. Ohayon. Mixed variational formulation of finite element analysis of acoustoelastic/slosh fluidstructure interaction. J of Fluids and Structures, 4:35-57, 1990.

[10] M.A. Hamdi. A displacement method for the analysis of vibrations of coupled fluid-structure systems. Int. J. Num. Meth, 13:139-150, 1978.

[11] C. Militello and C.A. Felippa. The Individual Element Test Revisited. In: Oate et al. Springer-Verlag, 1991.

[12] H. Morand and R. Ohayon. Substructure variational analysis of the vibrations of coupled fluid-structure system. finite element results. Int. J. Num. Meth. Eng, 14:741-755, 1979.

[13] L.G. Olson and K.J. Bathe. Analysis of fluid-structure interactions. a direct symmetric coupled formulation based on the fluid velocity potential. Computers \& Structures, 21:21-32, 1985.

[14] X. Wang and K.J. Bathe. Displacement/pressure based finite element formulations for acoustic fluid-structure interactions. In. J. Num. Meth. Engnr, 40:2011-2017, 1997.

[15] O.C. Zienkiewicz and R.L. Taylor. The finite element method Vol I:The Basis. Butterworth-Heinemann, Oxford, 2000. 